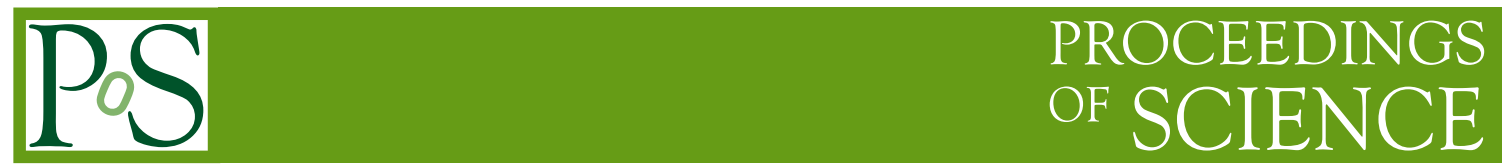

\title{
Leptonic decays of light hadronic states and rare $B$ decays
}

\author{
Aidan Grummer ${ }^{a, 1, *}$ \\ ${ }^{a}$ Department of Physics and Astronomy, University of New Mexico, \\ 210 Yale Blvd NE, Albuquerque, New Mexico, USA \\ E-mail: agrummer@unm.edu
}

Recent results in the study of leptonic decays of light hadronic states and rare $B$ decays are presented. Measurements of the properties of $B_{\mathrm{s}}^{0}$ mesons decaying to $\mu^{+} \mu^{-}$and searches for $B^{0}$ mesons decaying to $\mu^{+} \mu^{-}$by both the ATLAS and CMS collaborations are discussed. Probing lepton flavour violation via searches for $\tau$ leptons decaying to three muons with ATLAS and CMS is also discussed.

The Eighth Annual Conference on Large Hadron Collider Physics-LHCP2020 25-30 May, 2020

online

${ }^{1}$ on behalf of the ATLAS and CMS Collaborations

*Speaker 


\section{Introduction}

Flavour-changing neutral-current processes are highly suppressed in the Standard Model (SM). The branching fractions of the decays $B_{(\mathrm{s})}^{0} \rightarrow \mu^{+} \mu^{-}$are, in addition, helicity suppressed in the $\mathrm{SM}$, and are predicted to be $\mathcal{B}\left(B_{\mathrm{s}}^{0} \rightarrow \mu^{+} \mu^{-}\right)=(3.66 \pm 0.14) \times 10^{-9}$ and $\mathcal{B}\left(B^{0} \rightarrow \mu^{+} \mu^{-}\right)=$ $(1.03 \pm 0.05) \times 10^{-10}[1]$. The SM predicts that only the heavy mass eigenstate, with measured lifetime $\tau_{\mathrm{B}_{\mathrm{sH}}^{0}}=1.615 \pm 0.009 \mathrm{ps}$ [2], contributes to the $B_{\mathrm{s}}^{0} \rightarrow \mu^{+} \mu^{-}$decay. Significant deviations from SM predictions could arise in models involving non-SM heavy particles, including those predicted in the Minimal Supersymmetric Standard Model [3] and in extensions such as Minimal Flavour Violation [4] and Two-Higgs-Doublet Models [5].

Observing charged lepton flavour violation would be a major breakthrough in understanding the matter content of the universe. The branching fraction of $\tau \rightarrow 3 \mu$ is expected to be very small in the SM: $\mathcal{B}(\tau \rightarrow 3 \mu)<10^{-14}[6]$. Some extensions to the SM [7, 8] lead to a branching fraction orders of magnitude greater $\left(10^{-10}-10^{-8}\right)$, within reach of experimental confirmation.

The latest experimental results of the $B_{(\mathrm{s})}^{0} \rightarrow \mu^{+} \mu^{-}$analyses and the experimental searches for the lepton flavour violating decay $\tau \rightarrow 3 \mu$ with the ATLAS [9] and CMS [10] experiments are presented in this proceeding. The analyses discussed herein use data collected in $p p$ collisions at the CERN LHC.

\section{Measurements of the properties of $B_{\mathbf{s}}^{0} \rightarrow \mu^{+} \mu^{-}$and searches for $B^{0} \rightarrow \mu^{+} \mu^{-}$}

In the ATLAS $B_{(\mathrm{s})}^{0} \rightarrow \mu^{+} \mu^{-}$study [11], data corresponding to an effective integrated luminosity of $26.3 \mathrm{fb}^{-1}$ collected at a centre-of-mass energy $\sqrt{s}=13 \mathrm{TeV}$ in 2015 and 2016 of LHC Run 2 are combined with $25 \mathrm{fb}^{-1}$ collected at $\sqrt{s}=7$ and $8 \mathrm{TeV}$ in 2011 and 2012 of Run 1, respectively. In the CMS study [12], the data correspond to integrated luminosities of 5 and $20 \mathrm{fb}^{-1}$ recorded in 2011 and 2012 at $\sqrt{s}=7$ and $8 \mathrm{TeV}$, respectively, and $36 \mathrm{fb}^{-1}$ recorded in 2016 at $\sqrt{s}=13 \mathrm{TeV}$. Due to operational instabilities experienced with the CMS microstrip detector, CMS Run 2 data are divided into two separate running periods, denoted 2016A and 2016B. Data are further separated into the forward and central regions of the detector.

The notation used throughout this section refers to the combination of processes and their charge-conjugates, unless otherwise specified. In both studies the $B_{s}^{0} \rightarrow \mu^{+} \mu^{-}$and $B^{0} \rightarrow \mu^{+} \mu^{-}$ branching fractions are measured relative to the reference decay mode $B^{+} \rightarrow J / \psi\left(\rightarrow \mu^{+} \mu^{-}\right) K^{+}$ which is abundant and has a well-measured branching fraction $\mathcal{B}\left(B^{+} \rightarrow J / \psi K^{+}\right) \times \mathcal{B}(J / \psi \rightarrow$ $\left.\mu^{+} \mu^{-}\right)$. The $B^{0} \rightarrow \mu^{+} \mu^{-}\left(B_{s}^{0} \rightarrow \mu^{+} \mu^{-}\right)$branching fraction can be extracted as:

$$
\mathcal{B}\left(B_{(s)}^{0} \rightarrow \mu^{+} \mu^{-}\right)=\frac{N_{d(s)}}{\varepsilon_{\mu^{+} \mu^{-}}} \times\left[\mathcal{B}\left(B^{+} \rightarrow J / \psi K^{+}\right) \times \mathcal{B}\left(J / \psi \rightarrow \mu^{+} \mu^{-}\right)\right] \frac{\varepsilon_{J / \psi K^{+}}}{N_{J / \psi K^{+}}} \times \frac{f_{u}}{f_{d(s)}}
$$

where $N_{d}\left(N_{s}\right)$ is the $B^{0} \rightarrow \mu^{+} \mu^{-}\left(B_{s}^{0} \rightarrow \mu^{+} \mu^{-}\right)$signal yield, $N_{J / \psi K^{+}}$is the $B^{+} \rightarrow J / \psi K^{+}$reference channel yield, $\varepsilon_{\mu^{+} \mu^{-}}$and $\varepsilon_{J / \psi K^{+}}$are the corresponding values of acceptance times efficiency and $f_{u} / f_{d}\left(f_{u} / f_{s}\right)$ is the ratio of the hadronisation probabilities of a $b$-quark into $B^{+}$and $B^{0}\left(B_{s}^{0}\right)$.

The background to the $B_{(s)}^{0} \rightarrow \mu^{+} \mu^{-}$signal originates from three main sources: (1) the continuum background, the dominant combinatorial component, which consists of muons originating from uncorrelated hadron decays, (2) partially reconstructed decays where one or more of the 
final-state particles $(X)$ in a $b$ hadron decay are not reconstructed, and (3) the peaking background due to $B_{(\mathrm{s})}^{0} \rightarrow h h^{\prime}$ decays, with both hadrons misidentified as muons. In both analyses, a boosted decision tree (BDT) is implemented to suppress the dominant continuum background.

The $B^{+}$yield for the reference channel is extracted with an unbinned extended maximum likelihood fit to the $J / \psi K^{+}$invariant mass distribution. The fit includes four components: $B^{+} \rightarrow$ $J / \psi K^{+}$decays, Cabibbo-suppressed $B^{+} \rightarrow J / \psi \pi^{+}$decays, partially reconstructed $B$ decays $\left(B^{+} \rightarrow\right.$ $\left.J / \psi K^{+} X\right)$, and the continuum background composed mostly of $b \bar{b} \rightarrow J / \psi X$ decays.

The dimuon candidates are classified according to the BDT output in each experiment. The 2015-2016 ATLAS yield, determined from an unbinned maximum likelihood fit performed on the dimuon invariant mass distribution simultaneously across all BDT intervals, is $N_{s}=80 \pm 22$ and $N_{d}=-12 \pm 20$ compared to the SM expectation of $N_{s}=91$ and $N_{d}=10$. Summing over all data subset categories (separated by year and detector region), CMS observes a total $B_{s}^{0} \rightarrow \mu^{+} \mu^{-}$yield of $61_{-13}^{+15}$ candidates, consistent with SM expectations.

The branching fraction measurements for $B_{s}^{0} \rightarrow \mu^{+} \mu^{-}$and upper limits on $B^{0} \rightarrow \mu^{+} \mu^{-}$are, for the combined Run 1 and 2015-2016 Run 2 ATLAS data:

$$
\begin{aligned}
& \mathcal{B}\left(B_{s}^{0} \rightarrow \mu^{+} \mu^{-}\right)=\left(2.8_{-0.7}^{+0.8}\right) \times 10^{-9} \\
& \mathcal{B}\left(B^{0} \rightarrow \mu^{+} \mu^{-}\right)<2.1 \times 10^{-10} \text { at } 95 \% \mathrm{CL},
\end{aligned}
$$

and for all CMS data categories:

$$
\begin{aligned}
& \mathcal{B}\left(B_{s}^{0} \rightarrow \mu^{+} \mu^{-}\right)=[2.9 \pm 0.7(\exp ) \pm 0.2(\text { frag })] \times 10^{-9} \\
& \mathcal{B}\left(B^{0} \rightarrow \mu^{+} \mu^{-}\right)<3.6 \times 10^{-10} \text { at } 95 \% \mathrm{CL} .
\end{aligned}
$$

The likelihood contours of the fits are shown in Figure 1 for (left) ATLAS and (right) CMS, together with the SM expectation.

A two-dimensional unbinned maximum likelihood fit to the dimuon invariant mass and the proper decay time is implemented to extract the $B_{s}^{0} \rightarrow \mu^{+} \mu^{-}$effective lifetime in the CMS data. The fit includes the signal and each background component described above. The $B_{s}^{0} \rightarrow \mu^{+} \mu^{-}$lifetime is measured to be:

$$
\tau_{\mu^{+} \mu^{-}}=\left[1.70_{-0.43}^{+0.60}(\text { stat }) \pm 0.09(\text { syst })\right] \mathrm{ps},
$$

consistent with the SM expectation.

\section{Probing lepton flavour violation via searches for $\tau \rightarrow 3 \mu$ decays}

The ATLAS search for neutrinoless $\tau \rightarrow 3 \mu$ [13] utilizes $20.3 \mathrm{fb}^{-1}$ of data at $\sqrt{s}=8 \mathrm{TeV}$ collected in 2012. The CMS search [14] uses the data set collected in 2016 at $\sqrt{s}=13 \mathrm{TeV}$, corresponding to an integrated luminosity of $33 \mathrm{fb}^{-1}$.

The ATLAS search uses the source channel: $W \rightarrow \tau v$. A $\tau$-neutrino from the $W$ boson decay appears as missing transverse energy $\left(E_{\mathrm{T}}^{\mathrm{miss}}\right)$. The three muons from the $\tau$ lepton flavour violating decay are expected to have close geometric proximity. The branching fraction is calculated as:

$$
\mathcal{B}(\tau \rightarrow 3 \mu)=\frac{N_{s}}{\left(\mathcal{A} \times \epsilon_{S}\right) N_{W \rightarrow \tau \nu}},
$$



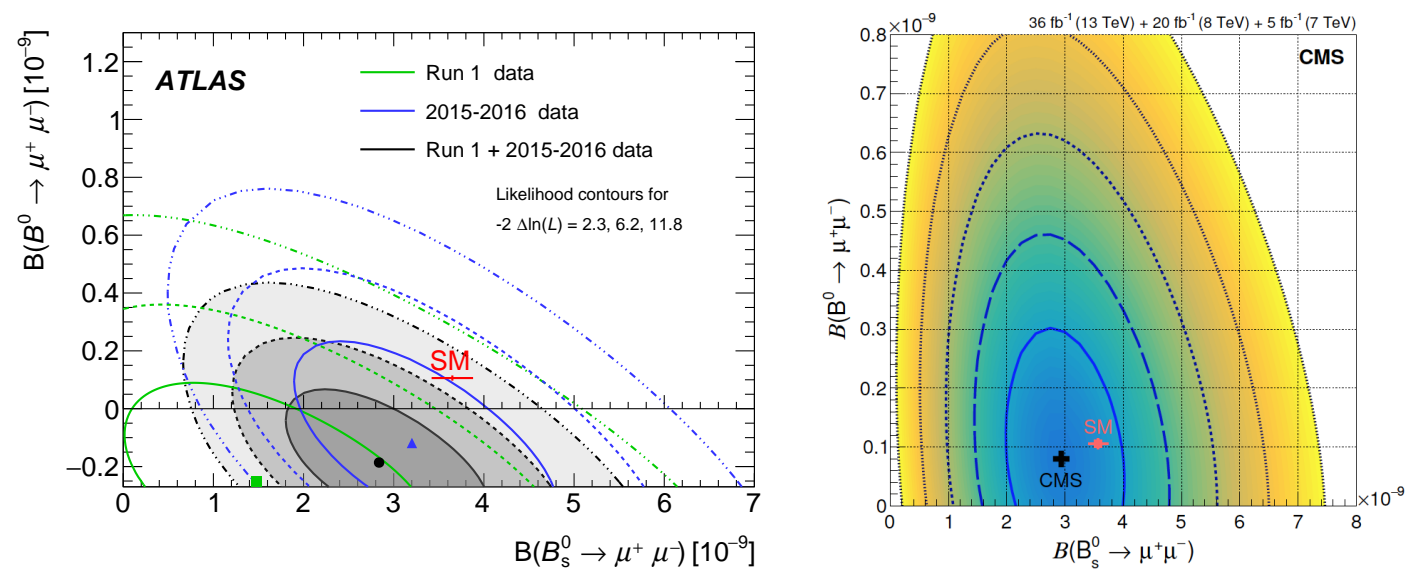

Figure 1: (Left) likelihood contours for the combination of the ATLAS Run 1 and 2015-2016 Run 2 results (shaded areas). The contours are obtained from the combined likelihoods of the two analyses, for values of $-2 \Delta \ln (\mathcal{L})$ equal to 2.3, 6.2 and 11.8. The contours for the individual Run 2 2015-2016 and Run 1 results are overlaid. The SM predictions and their uncertainties are included. Figure from [11]. (Right) likelihood contours for the fit to the CMS branching fractions $\mathcal{B}\left(B_{s}^{0} \rightarrow \mu^{+} \mu^{-}\right)$and $\mathcal{B}\left(B^{0} \rightarrow \mu^{+} \mu^{-}\right)$, together with the best-fit value (cross) and the SM expectation (solid square). The contours correspond to regions with 1-5 standard deviation coverage. Figure from [12].

where $N_{S}$ is the number of signal events, $\mathcal{A} \times \epsilon_{S}$ is the acceptance times efficiency of the signal and $N_{W \rightarrow \tau v}$ is the number of $\tau$ leptons produced via the decay $W \rightarrow \tau v$.

A BDT is trained to discriminate the background. The three-muon mass distribution of the ATLAS data is shown in Figure 2 (left) using tight selection criteria on the data (including selections on the three-muon vertex fit quality, $E_{\mathrm{T}}^{\text {miss }}$, mass restrictions for two muons with the same-charge and opposite-charge; further details can be found in [13]) paired with a loose BDT cut, $x_{0}$, used to remove background-like events, and a final BDT cut, $x_{1}$, optimizing the expected upper limit on the branching fraction. Figure 2 (left) also shows a fit to the sidebands as well as the simulated signal. The observed upper limit on the branching fraction in the ATLAS analysis is:

$$
\mathcal{B}(\tau \rightarrow 3 \mu)<3.76 \times 10^{-7} \text { at } 90 \% \mathrm{CL} .
$$

The CMS search exploits $\tau$ leptons produced in $D$ and $B$ meson decays. The branching fraction is measured using the normalisation channel: $D_{\mathrm{s}} \rightarrow \phi \pi \rightarrow \mu \mu \pi$. Data are separated into three categories based on the three-muon mass resolution. A BDT is trained using simulated signal data and the three-muon mass sidebands for each mass resolution category. Two BDT regions are optimized by maximizing the expected search sensitivity and used for signal extraction and uncertainty estimates. Figure 2 (right) shows the three-muon mass distribution for one of the three mass resolution categories and the corresponding highest BDT bin. The signal is normalised in each category, assuming a branching fraction $\mathcal{B}(\tau \rightarrow 3 \mu)=10^{-7}$. The upper limit on the branching fraction is:

$$
\mathcal{B}(\tau \rightarrow 3 \mu)<8.8 \times 10^{-8} \text { at } 90 \% \mathrm{CL}
$$



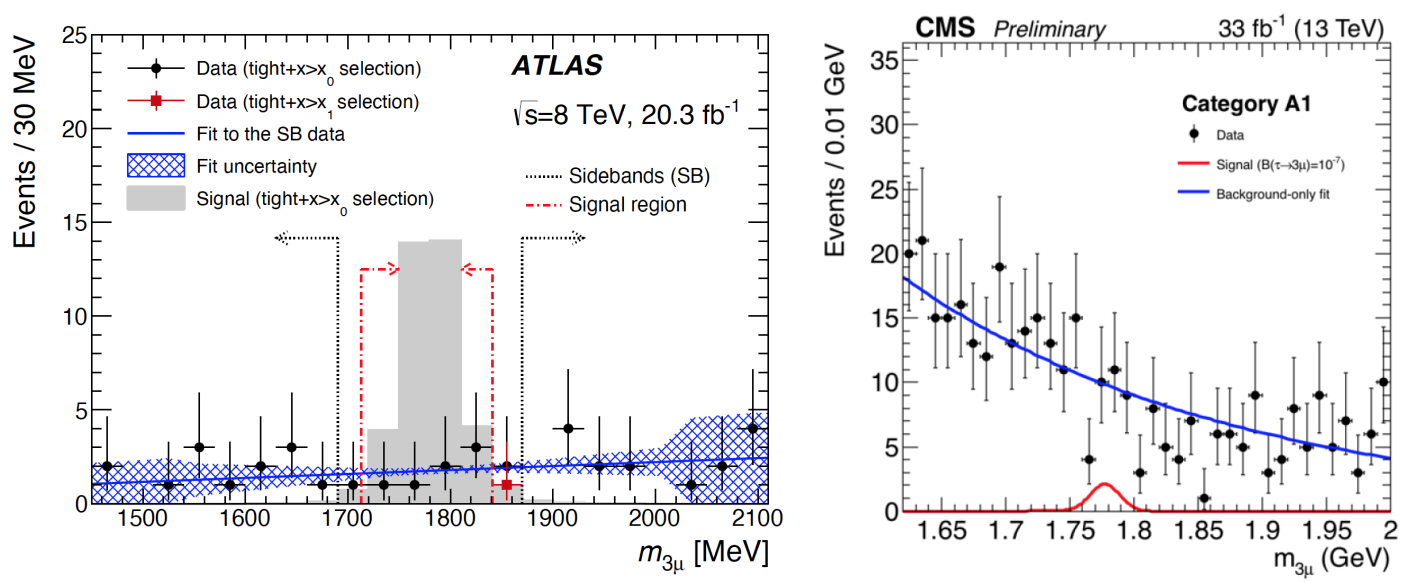

Figure 2: (Left) the three-muon mass distribution in the range [1450, 2110] MeV shown for the tight $+x>x_{0}$ selection by solid black circles and for the tight $+x>x_{1}$ selection by the solid red square. The sideband and signal regions are indicated by the arrows. The tight $+x>x_{0}$ data are fit in the two sidebands simultaneously, excluding the events in the blinded region. The hatched area shows the uncertainty in the fit due to the sideband range definition, the $x_{0}$ cut location and the fit function choice. The solid grey area shows the signal shape (obtained from simulation), normalised to the area of the data for the tight $+x>x_{0}$ selection. Figure from [13]. (Right) the three-muon mass distributions in one (A1) of six independent event categories described in the text. Data are shown with points. The background-only fit and the expected signal for $\mathcal{B}(\tau \rightarrow 3 \mu)=10^{-7}$ are shown with lines. Figure from [14].

\section{Conclusions}

Results of the studies performed by ATLAS and CMS of the rare decays of $B_{S}^{0}$ and $B^{0}$ mesons into $\mu^{+} \mu^{-}$have been presented. The results are compatible with the SM predictions. Searches for neutrinoless $\tau \rightarrow 3 \mu$ decays performed by ATLAS and CMS have been presented. No excess above the expected background is observed.

\section{References}

[1] M. Beneke, C. Bobeth, and R. Szafron, JHEP 10 (2019) 232.

[2] Particle Data Group collaboration, Phys. Rev. D 98 (2018) 030001.

[3] C.-S. Huang, W. Liao, and Q.-S. Yan, Phys. Rev. D 59 (1998) 011701.

[4] G. D’Ambrosio, G. F. Giudice, G. Isidori, and A. Strumia, Nucl. Phys. B 645 (2002) 155.

[5] K. S. Babu and C. F. Kolda, Phys. Rev. Lett. 84 (2000) 228.

[6] X.-Y. Pham, Eur. Phys. J. C 8 (1999) 513516.

[7] M. Raidal et al., Eur. Phys. J. C 57 (2008) 13182.

[8] W. J. Marciano, T. Mori, and J. M. Roney, Ann. Rev. Nucl. Part. Sci. 58 (2008) 315.

[9] ATLAS Collaboration, JINST 3 (2008) S08003. 
[10] CMS Collaboration, JINST 3 (2008) S08004.

[11] ATLAS Collaboration, JHEP 04 (2019) 098.

[12] CMS Collaboration, JHEP 04 (2020) 188.

[13] ATLAS Collaboration, Eur. Phys. J. C 76 (2016) 232.

[14] CMS Collaboration, CMS-PAS-BPH-17-004 (2019). 